\title{
Design of Software Defined Radio Platform Resource Model Based on SCA
}

\author{
Yang Yunlong, Lu Yourong, Cai Qian, Lai Yingyong \\ Southwest Electronics and Telecommunication Technology Research Institute \\ Chengdu, China \\ eric.yy@163.com
}

\begin{abstract}
Software Defined Radio (SDR) platform resource model is the description and organization of the SDR platform resource. It's the foundation of the SDR platform resource management. Through these years, the Software Communication Architecture (SCA) has becoming the most famous specification for SDR, which standardizes the description for the SDR platform resource. Aiming at the shortages of the resource model in SCA, this paper discusses a SDR platform resource model, proposes a transfer port model and a connection model, designs a nested data model for the SDR platform resource, and a plane graph model which is easy to analyze and process.
\end{abstract}

\section{Keywords-SDR; SCA; Resource Model}

\section{INTRODUCTION}

Along with the development of the high speed digital signal processing, Specialized Hardware Processor (SHP) such as DSP or FPGA is widely used in the SDR platform, which makes the SDR platform resource more various and complex. In order to realize different functions in the same SDR platform, the SDR platform must provide the resource flexibly for the different function software. The Software Communication Architecture (SCA) is developed by the US military Joint Tactical Radio System (JTRS) Joint Program Office (JPO) [1 3], which has becoming the most famous specification for SDR, and it provides a standardized logic model for the SDR platform resource.

SCA uses the Common Object Request Broker Architecture (CORBA) middleware to solve the problem of waveform portability on the General Purpose Processor (GPP), through which the distributed processors communicate with each other effectively, so the resource model in SCA is the logic model based on CORBA. But the CORBA is universally acknowledged that it is suitable for GPP and not for SHP. With the widely usage of SHP, the resource model in SCA is not enough to describe the SDR platform resource. How to create the SDR platform resource model has recently become a focus in the world.

The remainder of this paper is arranged as follows. Section 2 introduces the related work. Section 3 introduces the SDR platform resource model, which includes the date model and the graph model, and then discusses the translation from the data model to the graph model. The last section concludes the whole paper.

\section{RELATED WORK}

Many researchers have discussed the SDR platform resource model. Reference [4, 5] proposed a compute resource management framework, which regarded the SHP as compute resource, ignored the connections among the processors. Reference [6, 7] researched the dynamic deployment model for the SHP component, but the model could only describe the existence of the connections, and not distinguish the connection from others. This paper discusses the problems of the SDR platform resource model based on SCA, firstly designs a data model for the SDR platform resource to describe and organize the resource, then proposes a graph model which is easy to analyze, lastly discusses the translation from the data model to the graph model.

\section{SDR PLATFORM RESOURCE MODEL}

SDR platform resource model is the foundation of the SDR platform resource management. Aiming at the shortages of the resource model in SCA, this section designs a data model to describe and organize the SDR platform resource, and proposes a graph model to make the resource management easily.

\section{A. Data Model}

The SDR platform resource management model in SCA is a logic model based on the CORBA middleware, which is shown in figure 1 . The domain manager manages one or more device managers, and the device manager manages one or more devices. The domain manager can indirectly manage the devices which connect to the CORBA middleware through the device managers. The devices communicate with each other through the CORBA middleware, which makes the SDR platform resource logic model in SCA.

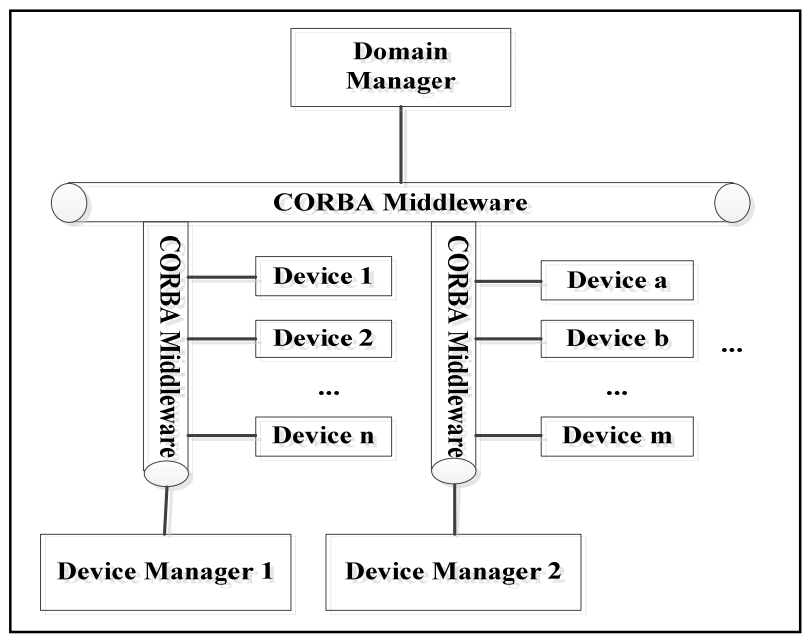

Figure 1. Resource management model in SCA. 
The logic model in SCA is not suitable for the SHP that could not run CORBA. Because of this, we couldn't regard the SHP as compute resource, and should consider the connections among the SHPs. Aiming at the problem we propose a transfer port model and a connection model for the connections, and design a nested data model for the SDR platform resource. The transfer port model is in figure 2 .

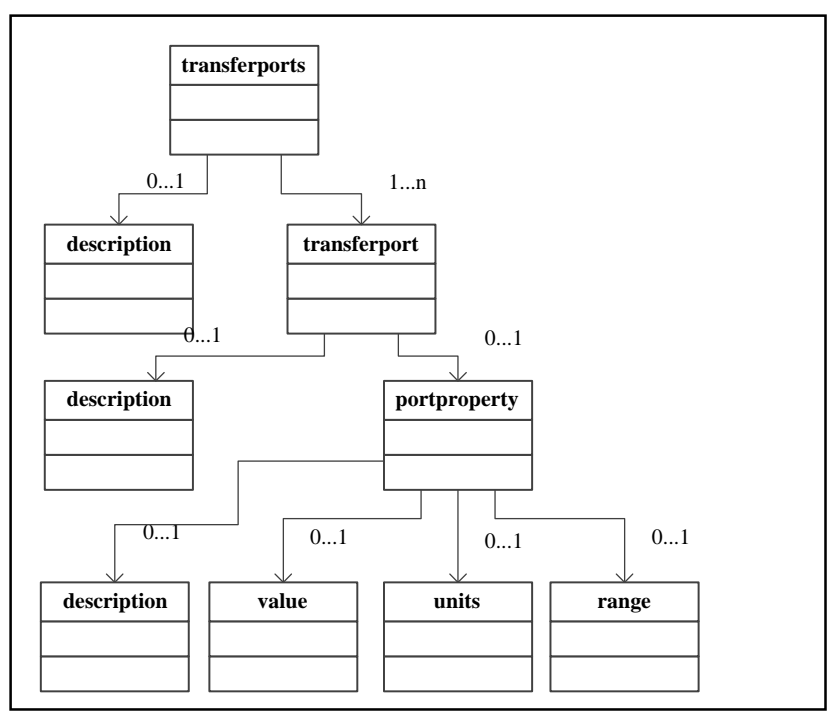

Figure 2. Transfer port model.

As shown in figure 2, the transfer port is the abstraction to the input or output end of the connection between two processors, it's the data interface of the processors. The attributes of the transfer port may contain the physical attribute or the protocol attribute, and we can describe them in the portproperty element. The transfer port can be instantiated to the port instantiation as the concrete port to the device. The port instantiation should indicate the port type, port direction and port position. The port instantiations of the same type could create connection to describe the connection between the processors. The connection is directional, and it may be bidirectional. We should describe the attributes of connection in the connectionproperty element.

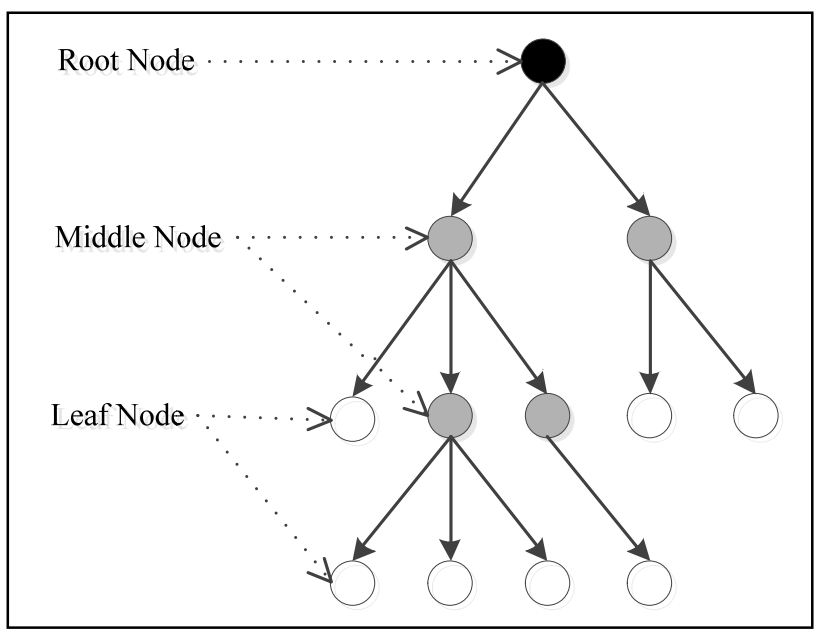

Figure 3. The architecture of the data model.

The architecture of the data model is shown as figure 3 . It's a nested architecture, and contains leaf node, middle node and root node from the bottom up. The leaf node is the bottom node, and can be instantiated to the node up to it. The middle node may be the all nodes between the leaf node and the root node. The root node is at the top, it can be regarded as a middle node and form the new root node up to it. The data model of each node is in figure 4 .

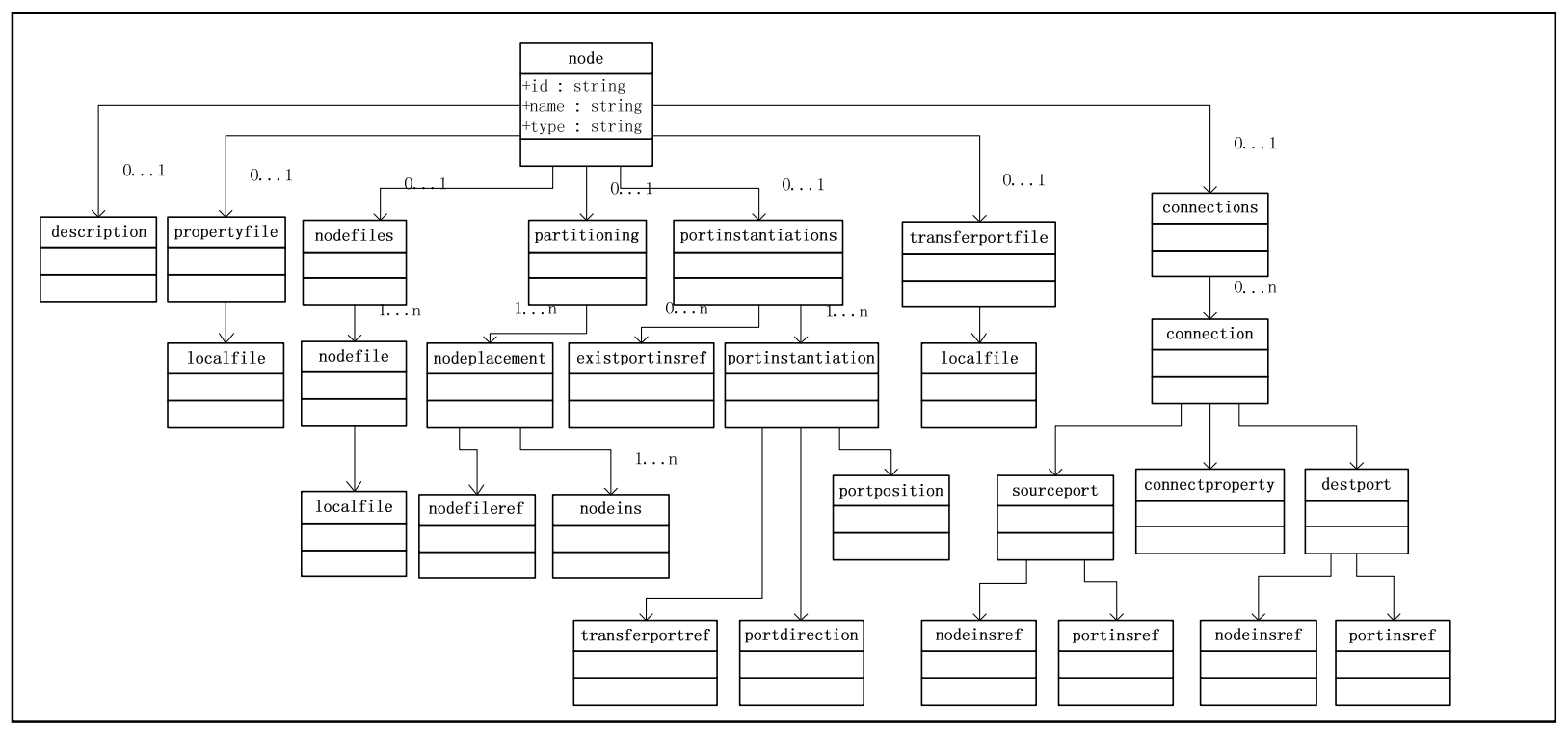

Figure 4. The data model of node. 
In the data model of node, the leaf node shall reference the property file in SCA in the propertyfile element to describe its attributes, and reference the transfer port file in the transferportfile element to describe the transfer port. The transfer port shall be instantiated to the port instantiation. The non-leaf node (which means the middle node and the root node) is comprised of the node instantiations which are instantiated by the sub-nodes. It shall reference the node file in the nodefiles element to describe the sub-nodes it contains, and describe the sub-node instantiations in the partitioning element. The port instantiation of the non-leaf node may reference the port instantiation of the sub-nodes, and the connection between sub-nodes should be described in the connections element.

\section{B. Graph Model}

It is very effective for the data model to describe and organize the SDR platform resource since the data model is flexible and convenient to expand. But it's hard to analyze or process with the data model such as the resource scheduling with it. So we design a graph model for the SDR platform resource, which is shown in figure 5 . The graph is directional, and the vertex stands for the device, the edge stands for the connection between the devices, the weight of edge stands for the attribute of the connection. The directional edge stands for the dataflow, it may be unidirectional or bidirectional. The edge between vertexes may be more than one, which means there are more than one dataflow between devices.

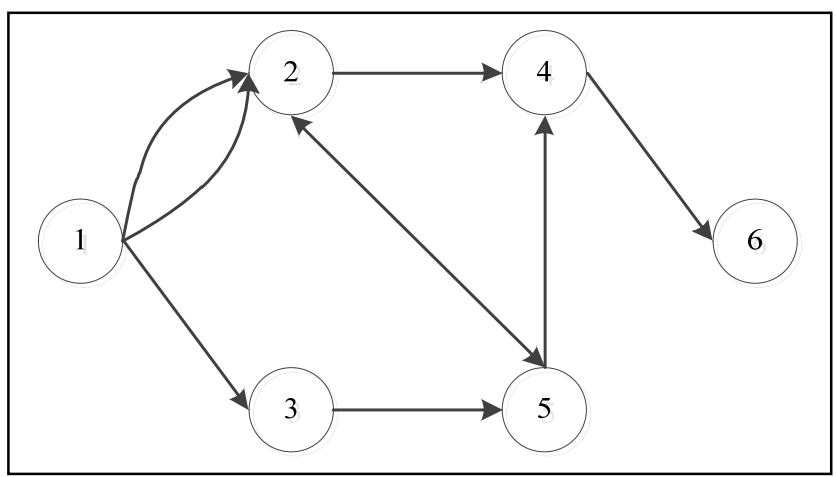

Figure 5. Graph model.

As the vertex in the graph stands for the device, we can describe the attribute of the device in the vertex. The edge in the graph stands for the connection between devices (or between two ports in other word), and the data flow from the source port to the destination port through the connection. We design the port-connection pair to describe the edge. In the source vertex we design the source port-connection pair, and in the destination vertex we design the destination portconnection pair. Each vertex can contain one or more portconnection pairs. In this way we describe the whole information in the vertex. The data structure of vertex in the graph model is shown in figure 6.

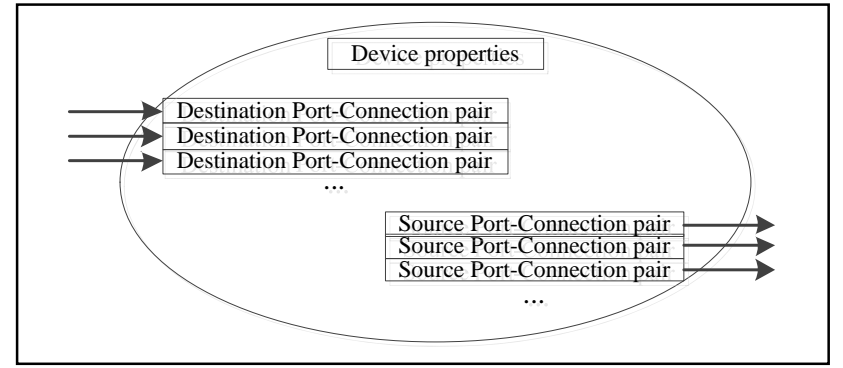

Figure 6. Data structure of vertex.

At last we should model the whole graph, and create the SDR platform resource graph model. We choose the adjacency list to present the whole graph, which is in figure 7 . The device list contains the all vertexes in the graph, and it's the source end for data. Each device has an adjacency list, and it's the destination end for data. Through creating the device list and the adjacency list, we describe the whole graph, and create the SDR platform resource graph model.

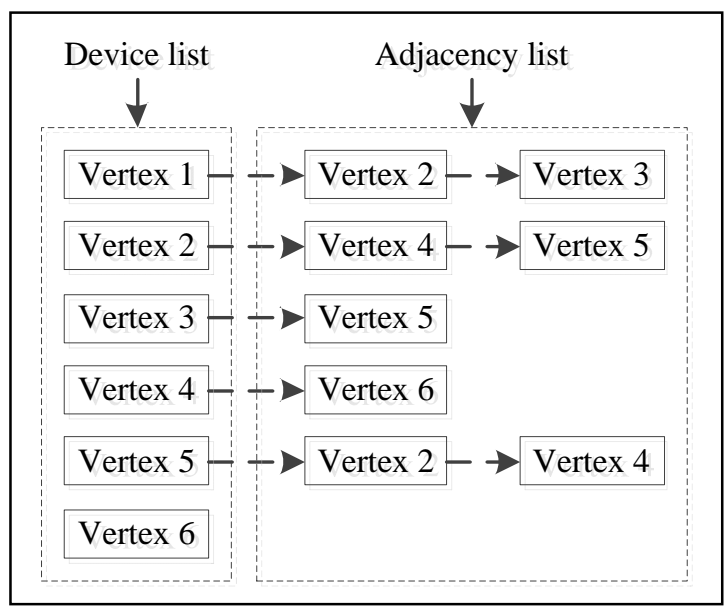

Figure 7. Adjacency list of the graph.

\section{Translation From Data Model To Graph Model}

It is very easy for the data model to describe and organize the SDR platform resource, but the data model is nested and layered. Each node in the data model represents a physical device, for example, leaf node represents the FPGA, DSP or CPU. When the leaf node is instantiated, it shall compose the node up to it, such as board, and so on. The graph model is a plane model. Each vertex in graph is relevant to the leaf node in the data model, and each edge is relevant to the connection between the leaf nodes. The translation flow from the data model to the graph model is shown in figure 8 . 


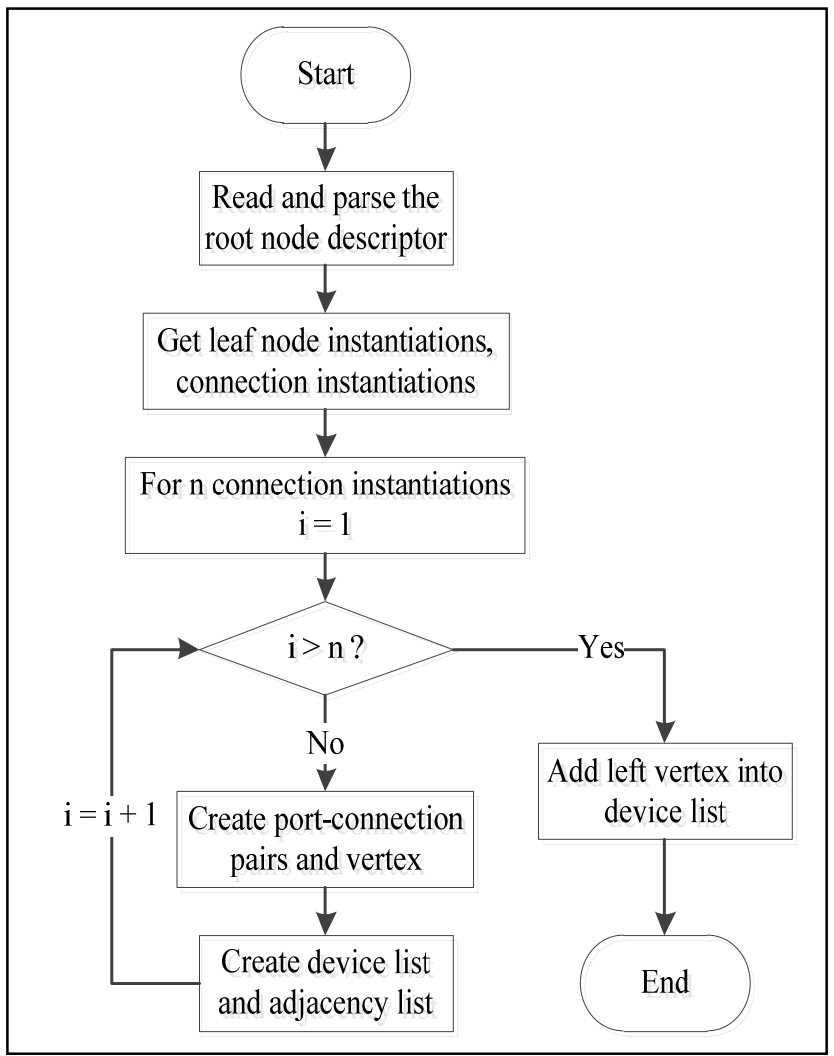

Figure 8. Translation flow from data model to graph model.

In order to stay the same with the descriptors in SCA, XML is used to create the data model elements. In figure 8, we get all the leaf node instantiations and the connection instantiations by parsing the root node descriptor. Each leaf node instantiation contains many transfer port instantiations, and each connection instantiation contains the source port and destination port with it. Then we create the portconnection pair according to the connection instantiation, and add it to the vertex port-connection pairs. Then we create the device list and the adjacency list. At last, we add the vertexes which have no connections into the device list.

\section{CONCLUSIONS}

This paper proposes a SDR platform resource model based on SCA. Firstly, this paper designs a transfer port model and connection model to describe the connection among the processors. Then, this paper designs a nested data model for SDR platform resource. In order to manage the SDR platform resource easily, this paper proposes a graph model for the SDR platform resource. At last, this paper discusses the translation from the data model to the graph model. Compared to the logic model in SCA, the resource model in this paper has some advantages: the data model describes the SDR platform resource more completed, and it's flexible and convenient to expand, the graph model is intuitional, and it's easy to analyze. The SDR platform resource model in this paper is useful, and can be used in the resource management of the SDR platform in future.

\section{REFERENCES}

[1] J. Mitola, SoftwareRadios: Survey, Critical Evaluation and Future Directions, proc. National Telesystems Conference, New York: IEEE Press, 1992.

[2] Neli Hayes, THE JTRS SCA SPECIFICATION $\cdots$ THE PAST, THE PRESENT, AND THE FUTURE, IEEE Military Communication Conference, May. 2005, pp. 2713-2719.

[3] JTRS JPEO, Software Communication Architecture Specification version 4.0, 2012.

[4] Vuk Marojevic, Xavier Reves Balleste, Antoni Gelonch, A Computing Resource Management Framework for Software-Defined Radios, IEEE TRANSACTIONS ON COMPUTERS, Oct. 2008, pp. 1399-1412.

[5] Ismael Gomez Miguelez, Vuk Marojevic, Antoni Gelonch Bosch, Resource Management For Software-defined Radio Clouds, IEEE Computer Society, Dec. 2012, pp. 44-53.

[6] Liu Qing, Shi Jun-wu, and Cao Kai, SHP Component Dynamic Deployment Scheme Based on SCA, Computer Engineering, Apr. 2012, pp. 227-229+232.

[7] Liu Qing, Cao Kai, and Xu Chun-lin, SHP component strategy deployment based on SCA, Modern Electronics Technique, Feb. 2012, pp. 77-80. 